\title{
Identification for Control: Some Results on Optimal Experiment Design
}

\author{
Urban Forssell and Lennart Ljung \\ Department of Electrical Engineering \\ Linkping University, S-581 83 Linkping, Sweden \\ WWW: http://www.control.isy.liu.se \\ Email: ufo@isy.liu.se, ljung@isy.liu.se
}

February 26, 1998

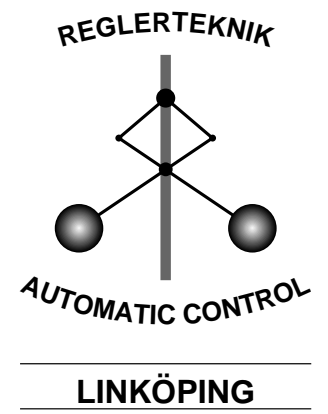

Report no.: LiTH-ISY-R-2014

Submitted to CDC '98

Technical reports from the Automatic Control group in Linkping are available by anonymous $\mathrm{ftp}$ at the address ftp.control.isy.liu.se. This report is contained in the compressed postscript file 2014.ps.Z. 


\title{
Identification for Control: Some Results on Optimal Experiment Design
}

\author{
Urban Forssell and Lennart Ljung \\ Division of Automatic Control \\ Department of Electrical Engineering \\ Linköping University, S-581 83 Linköping, Sweden \\ E-mail: ufo@isy.liu.se, ljung@isy.liu.se \\ URL: http://www.control.isy.liu.se/
}

February 26, 1998

\begin{abstract}
The problem of designing the identification experiments to make them maximally informative with respect to the intended use of the model is studied. A focus will be on model based control and we show how to choose the feedback regulator and the spectrum of the reference signal in case of closed-loop experiments. A main result is that when only the misfit in the dynamics model is penalized and when both the input and the output power are constrained then the optimal controller is given by the solution to a standard LQ problem. When only the input power is constrained, it is shown that openloop experiments are optimal. Some examples are also given to exemplify the theoretical results.
\end{abstract}

\section{Introduction}

The validation step in the identification procedure should be devoted to evaluating the model with respect to its intended use; simulation, prediction, control, etc. To make fair judgments it is important to know what factors affect the model's performance and how these factors can be manipulated. In this paper we focus on experiment design issues and especially how one should choose the feedback regulator in closed-loop experiments. The aim will be to design the experiments so as to minimize the variance errors in the identified models. This route was also taken in [2] and [4], and the presentation here will be inspired by these papers. See also [7].

The main drawback with the optimal solutions that will be derived is that they typically will depend on unknown quantities, like the unknown system that we are trying to identify. Clearly this will lead to infeasible designs. This problem is inevitable and shared with any optimal design problem. A way around this is to use iterations and to replace the true system in the optimal solutions with the current estimate of the system.

Despite this problem the optimal solutions derived here give important and useful insights into questions like "Should open-loop or closed-loop experiments be used?" and "To which frequency ranges should the input signal power be concentrated?". 
The rest of the presentation will unveil as follows. Section 2 contains some background material on the identification method used and the assumptions made on the underlying system. The notation will also be introduced in this section. In Section 3 we provide a motivation for the type of criterion we will consider. A key issue is that the criterion will reflect the variance error introduced when using identified models in the control design. Section 4 contains the main results and in Section 5 these results are applied to some well known control design schemes like internal model control and minimum variance control. Conclusions are given in Section 6.

\section{Preliminaries}

To evaluate a model's performance one needs some notion of the "best" or "true" model. We will assume that the true system is single-input single-output (SISO) and given by

$$
\begin{aligned}
& y(t)=G_{0}(q) u(t)+v(t) \\
& v(t)=H_{0}(q) e(t)
\end{aligned}
$$

Here $\{e(t)\}$ is white noise with variance $\lambda_{0}$. To simplify the notation we introduce the transfer matrix

$$
T_{0}(q)=\left[\begin{array}{l}
G_{0}(q) \\
H_{0}(q)
\end{array}\right]
$$

Given input-output data $Z^{N}=\{y(1), u(1), \ldots, y(N), u(N)\}$ and a parameterized model structure

$$
\hat{y}(t \mid \theta)=H^{-1}(q, \theta) G(q, \theta) u(t)+\left(1-H^{-1}(q, \theta)\right) y(t)
$$

we can estimate the model by the straightforward fit:

$$
\begin{aligned}
\hat{\theta}_{N} & =\arg \min _{\theta} V_{N}(\theta) \\
V_{N}(\theta) & =\sum_{t=1}^{N} \varepsilon^{2}(t, \theta) \\
\varepsilon(t, \theta) & =y(t)-\hat{y}(t \mid \theta)=H^{-1}(q, \theta)(y(t)-G(q, \theta) u(t))
\end{aligned}
$$

For more details on this identification method see, e.g., [7]. The model structure (3) may also be represented as

$$
y(t)=G(q, \theta) u(t)+H(q, \theta) e(t)
$$

In the sequel $G(q, \theta)$ and $H(q, \theta)$ will be called the dynamics model and the noise model, respectively. Let $\hat{G}_{N}(q)=G\left(q, \hat{\theta}_{N}\right)$ and $\hat{H}_{N}(q)=H\left(q, \hat{\theta}_{N}\right)$ and also introduce

$$
\hat{T}_{N}(q)=\left[\begin{array}{l}
\hat{G}_{N}(q) \\
\hat{H}_{N}(q)
\end{array}\right]
$$

The error between the true transfer matrix $T_{0}$ and the estimated one $\hat{T}_{N}$ will be denoted $\tilde{T}_{N}$,

$$
\tilde{T}_{N}(q)=T_{0}(q)-\hat{T}_{N}(q)=\left[\begin{array}{c}
\tilde{G}_{N}(q) \\
\tilde{H}_{N}(q)
\end{array}\right]
$$


with an obvious notation for $\tilde{G}_{N}$ and $\tilde{H}_{N}$. As the number of data tends to infinity and the model order $n$ increases we have that (cf. [5], [9])

$$
\operatorname{Cov} \hat{T}_{N}\left(e^{i \omega}\right) \approx \frac{n}{N} P(\omega)=\frac{n}{N} \Phi_{v}(\omega)\left[\begin{array}{cc}
\Phi_{u}(\omega) & \Phi_{u e}(\omega) \\
\Phi_{u e}(-\omega) & \lambda_{0}
\end{array}\right]^{-T}
$$

In this paper we will assume that the input is generated as

$$
u(t)=r(t)-K(q) y(t)
$$

and that the reference signal $r$ is uncorrelated with the disturbance $e$, i.e., that the cross spectrum $\Phi_{r e}=0$. We can then split the input spectrum into two parts, one that is due to $r$ and one that is due to $e$ :

$$
\Phi_{u}(\omega)=\Phi_{u}^{r}(\omega)+\Phi_{u}^{e}(\omega)
$$

By combining (1) and (10) we see that the input can be written

$$
u(t)=S_{0}(q) r(t)-K(q) S_{0}(q) H_{0}(q) e(t)
$$

Consequently

$$
\begin{aligned}
\Phi_{u}^{r}(\omega) & =\left|S_{0}\left(e^{i \omega}\right)\right|^{2} \Phi_{r}(\omega) \quad \Phi_{u}^{e}(\omega)=\left|K\left(e^{i \omega}\right)\right|^{2}\left|S_{0}\left(e^{i \omega}\right)\right|^{2}\left|H_{0}\left(e^{i \omega}\right)\right|^{2} \lambda_{0} \\
\Phi_{u e}(\omega) & =-K\left(e^{i \omega}\right) S_{0}\left(e^{i \omega}\right) H_{0}\left(e^{i \omega}\right) \lambda_{0}
\end{aligned}
$$

With these definitions we may rewrite $P$ as

$$
\begin{aligned}
P(\omega) & =\Phi_{v}(\omega)\left[\begin{array}{cc}
\Phi_{u}(\omega) & \Phi_{u e}(\omega) \\
\Phi_{u e}(-\omega) & \lambda_{0}
\end{array}\right]^{-T} \\
& =\frac{\Phi_{v}(\omega)}{\lambda_{0} \Phi_{u}(\omega)-\left|\Phi_{u e}(\omega)\right|^{2}}\left[\begin{array}{cc}
\lambda_{0} & -\Phi_{u e}(\omega) \\
-\Phi_{u e}(-\omega) & \Phi_{u}(\omega)
\end{array}\right]^{T} \\
& =\frac{\left|H_{0}\left(e^{i \omega}\right)\right|^{2}}{\Phi_{u}^{r}(\omega)}\left[\begin{array}{cc}
\lambda_{0} & -\Phi_{u e}(\omega) \\
-\Phi_{u e}(-\omega) & \Phi_{u}^{r}(\omega)+\Phi_{u}^{e}(\omega)
\end{array}\right]^{T}
\end{aligned}
$$

Here we have used the fact that

$$
\Phi_{v}(\omega)=\lambda_{0}\left|H_{0}\left(e^{i \omega}\right)\right|^{2}
$$

A remark regarding (15) is that this result holds not only for the direct method, considered here, but also for the so called indirect and joint input-output approaches to closed-loop identification. See, e.g., [3] and [1]. Therefore all the results below will also apply to these approaches as well.

For future use we also note that the output spectrum can be written

$$
\Phi_{y}(\omega)=\left|G_{0}\left(e^{i \omega}\right)\right|^{2} \Phi_{u}^{r}(\omega)+\left|S_{0}\left(e^{i \omega}\right)\right|^{2} \Phi_{v}(\omega)
$$




\section{Measuring the Performance Degradation}

Our focus here will be on model-based control. It is then natural to evaluate the model's performance by comparing the outputs of the closed-loop system resulting from control design using the "true" models and the estimated ones, respectively. In general this output will be a function of both $G$ and $H$ and some driving signal $w$ :

$$
y(t)=f(T(q)) w(t)
$$

The "true" or "best" signal is

$$
y_{0}(t)=f\left(T_{0}(q)\right) w(t)
$$

while the one resulting from the estimated models is

$$
\hat{y}_{N}(t)=f\left(\hat{T}_{N}(q)\right) w(t)
$$

A measure of the performance degradation can be obtained by computing the mean square error

$$
E \tilde{y}_{N}^{2}(t)=E\left(y_{0}(t)-\hat{y}_{N}(t)\right)^{2}
$$

Using first-order approximations we may write

$$
\tilde{y}_{N}(t)=\tilde{T}_{N}^{T}(q) F(q) w(t)
$$

where

$$
F(q)=\left.\frac{\partial}{\partial T} f(T)\right|_{T=T_{0}(q)}
$$

From the definition of the spectrum it follows that

$$
E \tilde{y}_{N}^{2}(t)=\frac{1}{2 \pi} \int_{-\pi}^{\pi} \Phi_{\tilde{y}_{N}}(\omega) d \omega=\frac{1}{2 \pi} \int_{-\pi}^{\pi} \operatorname{tr}[\Pi(\omega) C(\omega)] d \omega
$$

where

$$
\begin{aligned}
& \Pi(\omega)=E \tilde{T}_{N}\left(e^{-i \omega}\right) \tilde{T}_{N}^{T}\left(e^{i \omega}\right) \\
& C(\omega)=F\left(e^{i \omega}\right) \Phi_{w}(\omega) F^{T}\left(e^{-i \omega}\right)
\end{aligned}
$$

Thus, if we want to minimize the performance degradation due to the use of identified models, a natural criterion to minimize would be

$$
\bar{J}=\int_{-\pi}^{\pi} \operatorname{tr}[\Pi(\omega) C(\omega)] d \omega
$$

Let us comment on the matrix functions $\Pi$ and $C$. First, if the bias errors are negligible then $\Pi$ will be the (transpose of the) covariance matrix of the estimate $\hat{T}_{N}$,

$$
\Pi(\omega)=\left[\operatorname{Cov} \tilde{T}_{N}\left(e^{i \omega}\right)\right]^{T}
$$


The error made in this approximation should be small if the model structure is reasonably flexible. Using the standard result (9) we thus have

$$
\Pi(\omega) \approx \frac{n}{N} P^{T}(\omega)=\frac{n}{N} \Phi_{v}(\omega)\left[\begin{array}{cc}
\Phi_{u}(\omega) & \Phi_{u e}(\omega) \\
\Phi_{u e}(-\omega) & \lambda_{0}
\end{array}\right]^{-1}
$$

Second, the weighting function $C$ should reflect the relative importance of a good fit in $G$ and $H$, respectively, for different frequencies. We shall assume that $C$ is Hermitian and positive semi-definite as in (30). In general we may write

$$
C(\omega)=\left[\begin{array}{cc}
C_{11}(\omega) & C_{12}(\omega) \\
C_{12}(-\omega) & C_{22}(\omega)
\end{array}\right]
$$

If we neglect the scaling $n / N$ (which will have the affect that the prejudice against high order models is reduced), the criterion (27) simplifies to

$$
J=\int_{-\pi}^{\pi} \operatorname{tr}[P(\omega) C(\omega)] d \omega
$$

The route taken here follows the one in [2].

\section{Main Results}

In this section we will consider experiment design problems where the goal is to minimize $J$ with respect to the design variables, which we take as $K$ and $\Phi_{r}$. Other equivalent choices are also possible, e.g., $\Phi_{u}$ and $\Phi_{u e}$ or $K$ and $\Phi_{u}^{r}$. To make the designs realistic we will also impose constraints on the input power or the output power, or both. We will also discuss the case where power of the reference signal is bounded. Without loss of generality we will take the upper bounds to be unity. This is done to simplify the notation and can always be achieved by suitable scaling. In the sequel we also will omit the arguments $\omega$ and $e^{i \omega}$ whenever there is no risk of confusion.

First consider the case where penalty only is put on the misfit in $G$. This corresponds to $C_{12}=C_{22}=0$. We then have the following result which to our knowledge is new.

Theorem 1. The solution to the problem

$$
\min _{K, \Phi_{r}}\left\{\int_{-\pi}^{\pi} \operatorname{tr}[P C] d \omega: C_{12}=0, C_{22}=0 ; \int_{-\pi}^{\pi}\left\{\alpha \Phi_{u}+(1-\alpha) \Phi_{y}\right\} d \omega \leq 1, \alpha \in[0,1]\right\}
$$

is

$$
\begin{aligned}
K^{\text {opt }} & =\arg \min _{K} \int_{-\pi}^{\pi} \frac{\alpha|K|^{2}+(1-\alpha)}{\left|1+G_{0} K\right|^{2}} \Phi_{v} d \omega \\
\Phi_{r}^{\text {opt }} & =\mu \sqrt{\Phi_{v} C_{11}} \frac{\left|1+G_{0} K^{o p t}\right|^{2}}{\sqrt{\alpha+\left.(1-\alpha) G_{0}\right|^{2}}}
\end{aligned}
$$

where $\mu$ is a constant, adjusted so that

$$
\int_{-\pi}^{\pi}\left\{\alpha \Phi_{u}+(1-\alpha) \Phi_{y}\right\} d \omega=1
$$


Remark: It should be noted that equation (33), defining the optimal controller $K^{\text {opt }}$, is a standard LQ problem. If $G_{0}$ and $\Phi_{v}$ were known this could be easily solved to give an explicit expression for $K^{o p t}$.

Proof. Replace the design variables $K$ and $\Phi_{r}$ by the equivalent pair $K$ and $\Phi_{u}^{r}$. Then, by using (15), (11), and (17), problem (32) can be rewritten as

$$
\min _{K, \Phi_{u}^{r}}\left\{\int_{-\pi}^{\pi} \frac{\Phi_{v}}{\Phi_{u}^{r}} C_{11} d \omega: \int_{-\pi}^{\pi}\left\{\left(\alpha+(1-\alpha)\left|G_{0}\right|^{2}\right) \Phi_{u}^{r}+\frac{\alpha|K|^{2}+(1-\alpha)}{\left|1+G_{0} K\right|^{2}} \Phi_{v}\right\} d \omega \leq 1, \alpha \in[0,1]\right\}
$$

The criterion function is independent of $K$ hence the optimal controller $K^{o p t}$ can be found by solving the LQ problem

$$
\min _{K} \int_{-\pi}^{\pi} \frac{\alpha|K|^{2}+(1-\alpha)}{\left|1+G_{0} K\right|^{2}} \Phi_{v} d \omega
$$

(Here it is implicitly assumed that $y(t)=G_{0}(q) u(t)+v(t), u(t)=-K(q) y(t)$, and $\alpha \in[0,1]$.) This proves (33). Define the constant $\gamma$ as

$$
\gamma=1-\int_{-\pi}^{\pi} \frac{\alpha\left|K^{o p t}\right|^{2}+(1-\alpha)}{\left|1+G_{0} K^{o p t}\right|^{2}} \Phi_{v} d \omega
$$

Problem (36) now reads

$$
\min _{\Phi_{u}^{r}}\left\{\int_{-\pi}^{\pi} \frac{\Phi_{v}}{\Phi_{u}^{r}} C_{11} d \omega: \int_{-\pi}^{\pi}\left(\alpha+(1-\alpha)\left|G_{0}\right|^{2}\right) \Phi_{u}^{r} d \omega \leq \gamma\right\}
$$

This problem has the solution (cf. [7], p. 376)

$$
\Phi_{u}^{r}=\mu \sqrt{\frac{\Phi_{v} C_{11}}{\left(\alpha+(1-\alpha)\left|G_{0}\right|^{2}\right)}}
$$

where $\mu$ is a constant, adjusted so that

$$
\int_{-\pi}^{\pi}\left(\left(\alpha+(1-\alpha)\left|G_{0}\right|^{2}\right) \Phi_{u}^{r} d \omega=\gamma\right.
$$

or in other words so that

$$
\int_{-\pi}^{\pi}\left\{\alpha \Phi_{u}+(1-\alpha) \Phi_{y}\right\} d \omega=1
$$

Consequently the optimal $\Phi_{r}$ is

$$
\Phi_{r}^{o p t}=\mu \sqrt{\Phi_{v} C_{11}} \frac{\left|1+G_{0} K^{o p t}\right|^{2}}{\sqrt{\alpha+(1-\alpha)\left|G_{0}\right|^{2}}}
$$

which ends the proof. 
We stress that the optimal controller $K^{o p t}$ in (33) can easily be found by solving the indicated discrete-time LQ problem (if $G_{0}$ and $\Phi_{v}$ were known). Among other things this implies that the optimal controller $K^{o p t}$ is guaranteed to stabilize the closed-loop system and be linear, of the same order as $G_{0}$. This is a clear advantage over the results reported in, e.g., [4] and also over the results in Theorem 3 below. Furthermore, the optimal controller is independent of $C_{11}$ which also is quite interesting and perhaps somewhat surprising. This means that whatever weighting $C_{11}$ is used in the design criterion, it is always optimal to use the LQ regulator (33) in the identification experiment.

From Theorem 1 we also see that closed-loop experiments are optimal as long as the is a constraint on the output power, i.e., as long as $\alpha \neq 1$. If $\alpha=1$ then $K^{o p t}=0$ and the optimal input spectrum $\Phi_{u}^{o p t}\left(=\Phi_{r}^{o p t}\right)$ becomes

$$
\Phi_{u}^{o p t}=\mu \sqrt{\Phi_{v} C_{11}}
$$

This side result can in fact be strengthened [7]:

Theorem 2. The problem

$$
\min _{K, \Phi_{r}}\left\{\int_{-\pi}^{\pi} \operatorname{tr}[P C] d \omega: \int_{-\pi}^{\pi} \Phi_{u} d \omega \leq 1, C_{12}=0\right\}
$$

has the solution

$$
\begin{aligned}
K^{o p t} & =0 \quad \text { (open-loop operation) } \\
\Phi_{r}^{o p t} & =\Phi_{u}^{o p t}=\mu \sqrt{\Phi_{v} C_{11}}
\end{aligned}
$$

where $\mu$ is a constant, adjusted so that

$$
\int_{-\pi}^{\pi} \Phi_{u} d \omega=1
$$

Consider now the case where the power of the reference signal is constrained. The following theorem, which we believe is new, then states what the optimal controller and reference spectrum are. The expression for the optimal controller also holds for the case where the reference signal is fixed and pre-specified (which would be the case if data were collected under normal (controlled) operation of the plant).

Theorem 3. The problem

$$
\min _{K, \Phi_{r}}\left\{\int_{-\pi}^{\pi} \operatorname{tr}[P C] d \omega: \int_{-\pi}^{\pi} \Phi_{r} d \omega \leq 1\right\}
$$

has the solution

$$
\begin{aligned}
K^{o p t} & =-\frac{C_{11} G_{0}^{*}+C_{12} H_{0}^{*}}{C_{11}\left|G_{0}\right|^{2}+2 \operatorname{Re}\left(C_{12} G_{0} H_{0}^{*}\right)+C_{22}\left|H_{0}\right|^{2}} \\
\Phi_{r}^{o p t} & =\mu \sqrt{\frac{\Phi_{v} \cdot \operatorname{det} C \cdot\left|H_{0}\right|^{2}}{C_{11}\left|G_{0}\right|^{2}+2 \operatorname{Re}\left(C_{12} G_{0} H_{0}^{*}\right)+C_{22}\left|H_{0}\right|^{2}}}
\end{aligned}
$$

where $\mu$ is a constant, adjusted so that

$$
\int_{-\pi}^{\pi} \Phi_{r} d \omega=1
$$


Remark: Note that $K^{\text {opt }}$ in (50) only is a feasible design if $K^{\text {opt }}$ is proper and stabilizes the closed-loop system. Unfortunately no such guarantees can be given, which is a clear disadvantage compared to the result in Theorem 1. The optimal solutions derived in [4] suffer from the same type of problems, as mentioned earlier. To circumvent this problem it was suggested in [4] that the so called Youla parameterization [8] should be used. This idea could lead to interesting extensions of the results in Theorem 3 also, although we will not study it any further here.

Proof. Introduce the following square-root factorization of $C$ :

$$
C=\left[\begin{array}{cc}
C_{1} & C_{2} \\
0 & C_{3}
\end{array}\right]\left[\begin{array}{cc}
C_{1} & C_{2} \\
0 & C_{3}
\end{array}\right]^{*}
$$

Clearly we have the relations

$$
\begin{aligned}
& C_{11}=\left|C_{1}\right|^{2}+\left|C_{2}\right|^{2} \\
& C_{12}=C_{2} C_{3}^{*} \\
& C_{22}=\left|C_{3}\right|^{2}
\end{aligned}
$$

With $P$ given by (15) we have that

$$
\operatorname{tr}[P C]=C_{22}\left|H_{0}\right|^{2}+\Psi
$$

where

$$
\begin{aligned}
\Psi & =\frac{\Phi_{v}}{\Phi_{u}^{r}}\left[C_{11}+2 \operatorname{Re}\left(C_{12}^{*} K S_{0} H_{0}\right)+C_{22}|K|^{2}\left|S_{0}\right|^{2}\left|H_{0}\right|^{2}\right] \\
& =\frac{\Phi_{v}}{\Phi_{u}^{r}}\left[\left|C_{1}\right|^{2}+\left|C_{2}+C_{3} K S_{0} H_{0}\right|^{2}\right] \\
& =\frac{\Phi_{v}}{\Phi_{r}}\left[\left|C_{1}\left(1+K G_{0}\right)\right|^{2}+\left|C_{2}\left(1+K G_{0}\right)+C_{3} K H_{0}\right|^{2}\right] \\
& =\frac{\Phi_{v}}{\Phi_{r}}\left\|\left[\begin{array}{ll}
C_{1} & C_{2}
\end{array}\right]+K\left[\begin{array}{ll}
C_{1} G_{0} & C_{2} G_{0}+C_{3} H_{0}
\end{array}\right]\right\|^{2}
\end{aligned}
$$

There is no constraint on $K$ so to minimize the criterion function we should, at each frequency, choose $K$ as the least-squares estimate

$$
\begin{aligned}
K^{\text {opt }} & =-\frac{\left[\begin{array}{ll}
C_{1} & C_{2}
\end{array}\right]\left[\begin{array}{ll}
C_{1} G_{0} & C_{2} G_{0}+C_{3} H_{0}
\end{array}\right]^{*}}{\left\|\left[\begin{array}{ll}
C_{1} G_{0} & C_{2} G_{0}+C_{3} H_{0}
\end{array}\right]\right\|^{2}} \\
& =-\frac{\left(\left|C_{1}\right|^{2}+\left|C_{2}\right|^{2}\right) G_{0}^{*}+C_{2} C_{3}^{*} H_{0}^{*}}{\left(\left|C_{1}\right|^{2}+\left|C_{2}\right|^{2}\right)\left|G_{0}\right|^{2}+2 \operatorname{Re}\left(C_{2} C_{3}^{*} G_{0} H_{0}^{*}\right)+\left|C_{3}\right|^{2}\left|H_{0}\right|^{2}} \\
& =-\frac{C_{11} G_{0}^{*}+C_{12} H_{0}^{*}}{C_{11}\left|G_{0}\right|^{2}+2 \operatorname{Re}\left(C_{12} G_{0} H_{0}^{*}\right)+C_{22}\left|H_{0}\right|^{2}}
\end{aligned}
$$

With this choice of $K$ it can be shown (by straightforward but tedious calculations) that

$$
\left.\operatorname{tr}[P C]\right|_{K=K^{\text {opt }}}=C_{22}\left|H_{0}\right|^{2}+\frac{\Phi_{v}}{\Phi_{r}} \frac{\operatorname{det} C \cdot\left|H_{0}\right|^{2}}{C_{11}\left|G_{0}\right|^{2}+2 \operatorname{Re}\left(C_{12} G_{0} H_{0}^{*}\right)+C_{22}\left|H_{0}\right|^{2}}
$$


The first term is independent of $\Phi_{r}$, hence we get (cf. [7], p. 376)

$$
\Phi_{r}^{o p t}=\mu \sqrt{\frac{\Phi_{v} \cdot \operatorname{det} C \cdot\left|H_{0}\right|^{2}}{C_{11}\left|G_{0}\right|^{2}+2 \operatorname{Re}\left(C_{12} G_{0} H_{0}^{*}\right)+C_{22}\left|H_{0}\right|^{2}}}
$$

where $\mu$ is a constant, adjusted so that

$$
\int_{-\pi}^{\pi} \Phi_{r}^{o p t} d \omega=1
$$

This proves the theorem.

Note that if $C$ is singular the optimal $\Phi_{r}$ in (51) can be chosen at will, as long as

$$
\int_{-\pi}^{\pi} \Phi_{r}^{o p t} d \omega=1
$$

This is due to the factor $\operatorname{det} C$ in (51) which vanishes when $C$ is singular. Furthermore, when $C$ is singular the optimal $K$ in (50) simplifies to

$$
K^{\text {opt }}=-\frac{C_{12}}{C_{12} G_{0}+C_{22} H_{0}}
$$

We have thus established the following theorem which is a variant of a result given in [6]. See also [2].

Theorem 4. If $C$ is singular then the problem

$$
\min _{K, \Phi_{r}}\left\{\int_{-\pi}^{\pi} \operatorname{tr}[P C] d \omega: \text { any constraint }\right\}
$$

has the solution

$$
\begin{aligned}
& K^{\text {opt }}=-\frac{C_{12}}{C_{12} G_{0}+C_{22} H_{0}} \\
& \Phi_{r}^{\text {opt }} \quad \text { arbitrary }
\end{aligned}
$$

as long as u given by

$$
u(t)=r(t)+\frac{C_{12}(q)}{C_{12}(q) G_{0}(q)+C_{22}(q) H_{0}(q)} y(t)
$$

is an admissible input.

\section{$5 \quad$ Examples}

\subsection{Internal Model Control}

In Internal Model Control (IMC) the control law is chosen as

$$
u(t)=K(q)\left(r_{c}(t)-y(t)\right)=\frac{Q(q)}{1-G(q) Q(q)}\left(r_{c}(t)-y(t)\right)
$$


where $Q$ is some stable transfer function and $G$ a model of the true plant $G_{0}$. Here $r_{c}$ denotes the reference signal to be used in the control application, not to be mixed up with the reference signal $r$ used in the identification experiment. Note that the control design will not depend on the noise model. Also note that the designed closed-loop system is

$$
G_{c}^{\text {des }}(q)=\frac{G(q) K(q)}{1+G(q) K(q)}=G(q) Q(q)
$$

For this 1-dof regulator $G_{c}$ coincides with the complementary sensitivity function. From (74) we see that $Q=G_{c} / G$ should be large where a raise in the system's gain is desired.

Ideally, that is when $G=G_{0}$, the "best" or "true" IMC controller results:

$$
K_{0}(q)=\frac{Q(q)}{1-G_{0}(q) Q(q)}
$$

In general the model $G$ is obtained through identification using $N$ data samples. In that case $G=\hat{G}_{N}$ and the corresponding controller is

$$
\hat{K}_{N}(q)=\frac{Q(q)}{1-\hat{G}_{N}(q) Q(q)}
$$

Neglecting the response from the noise source $e$ we obtain the "true" output $y_{0}$ as

$$
y_{0}(t)=\frac{G_{0}(q) K_{0}(q)}{1+G_{0}(q) K_{0}(q)} r_{c}(t)=G_{0}(q) Q(q) r_{c}(t)
$$

The actual output resulting from use of the suboptimal controller (76) is

$$
\hat{y}_{N}(t)=\frac{G_{0}(q) \hat{K}_{N}(q)}{1+G_{0}(q) \hat{K}_{N}(q)} r_{c}(t)=\frac{G_{0}(q) Q(q)}{1+\tilde{G}_{N}(q) Q(q)} r_{c}(t)
$$

A measure of the performance degradation can be obtained by studying the error signal $\tilde{y}_{N}=y_{0}-\hat{y}_{N}$. We have

$$
\begin{aligned}
\tilde{y}_{N}(t) & =G_{0}(q) Q(q)\left[1-\frac{1}{1+\tilde{G}_{N}(q) Q(q)}\right] r_{c}(t) \\
& =\frac{\tilde{G}_{N}(q)}{1+\tilde{G}_{N}(q) Q(q)} G_{0}(q) Q^{2}(q) r_{c}(t)
\end{aligned}
$$

Using first-order approximations we get

$$
\tilde{y}_{N}(t) \approx \tilde{G}_{N}(q) G_{0}(q) Q^{2}(q) r_{c}(t)
$$

and it follows that the resulting weighting matrix $C$ in this case is given by

$$
C_{I M C}=\left[\begin{array}{cc}
\left|G_{0}\right|^{2}|Q|^{4} \Phi_{r_{c}} & 0 \\
0 & 0
\end{array}\right]
$$

Suppose now that we want to solve the problem

$$
\min _{K, \Phi_{r}}\left\{\bar{E} \tilde{y}_{N}^{2}(t): \int_{-\pi}^{\pi}\left\{\Phi_{u}+\Phi_{y}\right\} d \omega<\infty\right\}
$$


where $K$ and $r$ refers to the set-up (10) used in the identification. Theorem 1 then states that the optimal strategy is to use closed-loop experiments with $K$ and $\Phi_{r}$ chosen as

$$
\begin{aligned}
& K^{\text {opt }}=\arg \min _{K} \int_{-\pi}^{\pi} \frac{|K|^{2}+1}{\left|1+G_{0} K\right|^{2}} \Phi_{v} d \omega \\
& \Phi_{r}^{\text {opt }} \propto \sqrt{\Phi_{v} \Phi_{r_{c}}} \cdot\left|G_{0}\right| \cdot|Q|^{2} \cdot \frac{\left|1+G_{0} K^{o p t}\right|^{2}}{\sqrt{1+\left|G_{0}\right|^{2}}}
\end{aligned}
$$

A remark on this choice of reference spectrum is that (for fixed $G_{0}$ and $\Phi_{v}$ ) $\Phi_{r}^{o p t}$ will be large where either of $\Phi_{r_{c}}$ or $|Q| \approx\left|G_{c}^{\text {des }} / G_{0}\right|$, is large.

\subsection{Generalized Minimum Variance Control}

In Generalized Minimum Variance Control (GMVC) the goal is to make the closed-loop system behave like

$$
y_{0}(t)=R(q) e(t)
$$

This can be accomplished by using a regulator of the form

$$
u(t)=-\frac{H_{0}(q)-R(q)}{G_{0}(q) R(q)} y(t)
$$

It can be shown that the degradation signal $\tilde{y}_{N}$ can be written (neglecting errors of order higher than 2)

$$
\tilde{y}_{N}(t)=\left[\begin{array}{ll}
\tilde{G}_{N}(q) & \tilde{H}_{N}(q)
\end{array}\right]\left[\begin{array}{c}
\frac{H_{0}(q)-R(q)}{G_{0}(q)} \\
-1
\end{array}\right] \frac{R(q)}{H_{0}(q)} e(t)
$$

leading to a $C$-matrix of the form

$$
C_{G M V C}=\lambda_{0}\left|\frac{R}{H_{0}}\right|^{2}\left[\begin{array}{cc}
\frac{\left|H_{0}-R\right|^{2}}{\left|G_{0}\right|^{2}} & -\frac{H_{0}-R}{G_{0}} \\
-\frac{H_{0}^{*}-R^{*}}{G_{0}^{*}} & 1
\end{array}\right]
$$

Note that $C_{G M V C}$ is singular. Hence the choice

$$
K_{y}^{o p t}=-\frac{-\left(H_{0}-R\right) / G_{0}}{-\left(H_{0}-R\right)+H_{0}}=\frac{H_{0}-R}{G_{0} R}
$$

minimizes

$$
\bar{E} \tilde{y}_{N}^{2}(t)
$$

subject to any constraint according to Theorem 4. In other words, the optimal controller is the true GMVC controller. This was first shown in [2]. 


\subsection{Model Reference Control}

In Model Reference Control (MRC) the closed-loop system should ideally behave like

$$
y_{0}(t)=Q(q) r_{c}(t)+R(q) e(t)
$$

for some desired $Q$ and $R$. This can be achieved by letting

$$
u(t)=K_{r, 0}(q) r_{c}(t)-K_{y, 0}(q) y(t)
$$

where

$$
\begin{aligned}
K_{r, 0}(q) & =\frac{Q(q) H_{0}(q)}{R(q) G_{0}(q)} \\
K_{y, 0}(q) & =\frac{H_{0}(q)-R(q)}{G_{0}(q) R(q)}
\end{aligned}
$$

Note that $Q=0$ would give us back the GMVC solution. When using identified models $\hat{G}_{N}$ and $\hat{H}_{N}$ in the control design we will obtain

$$
\begin{aligned}
\hat{K}_{r, N}(q) & =\frac{Q(q) \hat{H}_{N}(q)}{R(q) \hat{G}_{N}(q)} \\
\hat{K}_{y, N}(q) & =\frac{\hat{H}_{N}(q)-R(q)}{\hat{G}_{N}(q) R(q)}
\end{aligned}
$$

The corresponding sub-optimal output becomes

$$
\begin{aligned}
\hat{y}_{N}(t) & =\frac{G_{0}(q) \hat{K}_{r, N}(q)}{1+G_{0}(q) \hat{K}_{y, N}(q)} r_{c}(t)+\frac{H_{0}(q)}{1+G_{0}(q) \hat{K}_{y, N}(q)} e(t) \\
& =\frac{G_{0}(q) Q(q) \hat{H}_{N}(q)}{G_{0}(q) \hat{H}_{N}(q)-\tilde{G}_{N}(q) R(q)} r_{c}(t)+\frac{\hat{G}_{N}(q) R(q) H_{0}(q)}{G_{0}(q) \hat{H}_{N}(q)-\tilde{G}_{N}(q) R(q)} e(t)
\end{aligned}
$$

The degradation signal $\tilde{y}_{N}=y_{0}-\hat{y}_{N}$ is given by

$$
\begin{aligned}
\tilde{y}_{N}(t)=Q(q)\left[1-\frac{G_{0}(q) \hat{H}_{N}(q)}{G_{0}(q) \hat{H}_{N}(q)-\tilde{G}_{N}(q) R(q)}\right] & r_{c}(t) \\
+R(q) & {\left[1-\frac{\hat{G}_{N}(q) H_{0}(q)}{G_{0}(q) \hat{H}_{N}(q)-\tilde{G}_{N}(q) R(q)}\right] e(t) }
\end{aligned}
$$

Working out the algebra we get

$$
\begin{aligned}
\tilde{y}_{N}(t) & =-\frac{R(q)}{H_{0}(q)} \frac{\left\{\tilde{G}_{N}(q)\left[\frac{Q(q)}{G_{0}(q)} r_{c}(t)-\frac{H_{0}(q)-R(q)}{G_{0}(q)} e(t)\right]+\tilde{H}_{N}(q) e(t)\right\}}{\left(1-\left(\frac{\tilde{H}_{N}}{H_{0}}+\frac{\tilde{G}_{N} R}{G_{0} H_{0}}\right)\right)} \\
& \approx-\frac{R(q)}{H_{0}(q)}\left\{\tilde{G}_{N}(q)\left[\frac{Q(q)}{G_{0}(q)} r_{c}(t)-\frac{H_{0}(q)-R(q)}{G_{0}(q)} e(t)\right]+\tilde{H}_{N}(q) e(t)\right\}
\end{aligned}
$$


leading to

$$
C_{M R C}=\left|\frac{R}{H_{0}}\right|^{2}\left[\begin{array}{cc}
\frac{|Q|^{2}}{\left|G_{0}\right|^{2}} \Phi_{r_{c}}+\frac{\left|H_{0}-R\right|^{2}}{\left|G_{0}\right|^{2}} \lambda_{0} & -\frac{H_{0}-R}{G_{0}} \lambda_{0} \\
-\frac{H_{0}^{*}-R^{*}}{G_{0}^{*}} \lambda_{0} & \lambda_{0}
\end{array}\right]
$$

Note that

$$
C_{M R C}=\left|\frac{R}{H_{0}}\right|^{2}\left[\begin{array}{cc}
\frac{|Q|^{2}}{\left|G_{0}\right|^{2}} \Phi_{r_{c}} & 0 \\
0 & 0
\end{array}\right]+C_{G M V C}
$$

Consider now the design problem

$$
\min _{K, \Phi_{r}}\left\{\int_{-\pi}^{\pi} \operatorname{tr}\left[P C_{M R C}\right] d \omega: r(t)=r_{c}(t)\right\}
$$

where $K$ and $r$ refers to (10). The constraint that $r=r_{c}$ means that the identification experiment should be performed using the same reference signal as in the control application, or in other words that the data should be collected under normal operation of the controlled plant.

Using the results of Theorem 3 it can be shown that the optimal controller is

$$
K^{o p t}=-\frac{1}{G_{0}} \frac{|Q|^{2} \Phi_{r_{c}}+\left(|R|^{2}-H_{0} R^{*}\right) \lambda_{0}}{|Q|^{2} \Phi_{r_{c}}+|R|^{2} \lambda_{0}}=-\frac{1}{G_{0}}\left(1-\frac{H_{0} R^{*}}{|Q|^{2} \Phi_{r_{c}} / \lambda_{0}+|R|^{2}}\right)
$$

It is interesting to study how the optimal solution changes as a function of the control design variables $Q, R, \Phi_{r_{c}}$ and $\lambda_{0}$. Here we consider the following special cases:

- When $\Phi_{r_{c}} / \lambda_{0} \rightarrow \infty$,

$$
K^{o p t} \rightarrow-\frac{1}{G_{0}}
$$

We recognize the right-hand side expression as the optimal controller when $C_{12}=0$, $C_{22}=0$, cf. (50).

- When $\Phi_{r_{c}} / \lambda_{0} \rightarrow 0$,

$$
K^{o p t} \rightarrow-\frac{|R|^{2}-H_{0} R^{*}}{G_{0}|R|^{2}}=\frac{H_{0}-R}{G_{0} R}=K_{y, 0}
$$

The true MRC feedback controller $K_{y, 0}$ is thus optimal in the identification only when $\Phi_{r_{c}} / \lambda_{0}=0$. This is consistent with the result for GMVC since when there is no reference signal present the MRC scheme is equivalent to GMVC. The result also applies to the case $Q=0$.

\section{Conclusions}

We have studied the problem of optimal experiment design. A main result was that we, possibly for the first time, have been able to give explicit solution to a experiment design problem with constraints on both the input and output power. The optimal controller is in this case given by the solution to a standard LQ problem. Another main conclusion is that closed-loop experiments are optimal whenever the output variance is constrained, otherwise open-loop experiments are preferable. 


\section{References}

[1] U. Forssell and L. Ljung. Closed-loop identification revisited. Technical Report LiTH-ISYR-1959, Department of Electrical Engineering, Linköping University, Linköping, Sweden, 1997.

[2] M. Gevers and L. Ljung. Optimal experiment design with respect to the intended model application. Automatica, 22:543-554, 1986.

[3] M. Gevers, L. Ljung, and P. Van den Hof. Asymptotic variance expression for closed-loop identification and their relevance in identification for control. In Proceedings of the 11th IFAC Symposium on System Identification, volume 3, pages 1449-1454, Fukuoka, Japan, 1997.

[4] H. Hjalmarsson, M. Gevers, and F. De Bruyne. For model-based control design, closed loop identification gives better performance. Automatica, 32, 1996.

[5] L. Ljung. Asymptotic variance expressions for identified black-box transfer function models. IEEE Transactions on Automatic Control, 30(9):834-844, 1985.

[6] L. Ljung. Parametric methods for identification of transfer functions of linear systems. In C. L. Leondes, editor, Advances in Control, volume 24. Academic Press, 1986.

[7] L. Ljung. System Identification: Theory for the User. Prentice-Hall, 1987.

[8] M. Vidyasagar. Control System Synthesis: A Factorization Approach. MIT Press, 1985.

[9] Y.-C. Zhu. Black-box identification of MIMO transfer functions: asymptotic properties of prediction error models. International Journal of Adaptive Control and Signal Processing, 3:357-373, 1989. 